\title{
Singularity Loci of Spherical Parallel Mechanisms
}

\author{
Ilian A. Bonev \\ Department of Automated Manufacturing Engineering \\ École de technologie supérieure \\ 1100 Notre-Dame Street West, Montreal, QC, Canada H3C 1K3 \\ ilian.bonev@etsmtl.ca
}

\author{
Clément M. Gosselin \\ Department of Mechanical Engineering \\ Université Laval \\ Quebec City, QC, Canada G1K 7P4 \\ gosselin@gmc.ulaval.ca
}

\begin{abstract}
This paper presents the computation and representation of the Type 2 singularity loci of symmetric spherical parallel mechanisms based on a not-well-known intuitive orientation representation. The latter, previously introduced under the name of the Tilt-and-Torsion angles, is briefly described. Then, to illustrate the approach, the two most basic spherical parallel mechanisms are considered and their Type 2 singularities are fully analyzed for various designs.
\end{abstract}

Index Terms - spherical parallel mechanisms, wrists, singularities, orientation representation, Euler angles.

\section{INTRODUCTION}

Recently, there has been a certain breakthrough in the synthesis of spherical parallel mechanisms (SPMs), accomplished mainly by a couple of researchers in the field [1][3]. This completes a series of older works on more basic 3-DOF SPMs [4]-[6]. Still, there are most certainly new SPMs to be discovered, yet the design and analysis of even the most basic SPMs are far from complete.

One of the least studied problems related to SPMs is their singularity analysis or, more specifically, finding their singularity loci. For example, even though the prototype of the Agile Eye (a 3-DOF 3-RRR ${ }^{1}$ SPM with orthogonal joint axes) was built in 1993 [7], it was not until nearly a decade later that its singularities were fully studied [8]. Yet, how can one adopt a specific design without exact knowledge of its singularity-free workspace.

The only other works that focus on the Type 2 singularity loci $^{2}$ of SPMs (of 3-U $\underline{P} S / S$ type) are presented in [10] and [11]. In these works, analytic expressions for the singularity loci are obtained in the $Z Y Z$ and $X Y Z$ Euler angles but are very hard to interpret. The works illustrate clearly why the singularity loci of SPMs are so unattractive to study-it is not intuitive to represent them. By offering a geometric tool for computing and representing the properties of SPMs, we overcome this difficulty.

In the following section, we describe this geometric tool that should become a standard for the orientation representation of symmetric SPMs. Then, in Section III, we apply this tool for the computation and representation of the singularity loci of 3-U $\underline{P} S / S$ symmetric SPMs. Similarly, in Section IV, we analyze 3- $\underline{R R R}$ symmetric SPMs. Finally, a conclusion is given in Section V.

\footnotetext{
${ }^{1}$ It is common to denote parallel mechanisms by using the symbols $U$, $R, S$, and $P$, which stand respectively for universal, revolute, spherical, and prismatic joint. When a joint is actuated, its symbol is underlined.

${ }^{2}$ It is common to classify the singularities of parallel mechanisms into two types [9]: Type 1 (or serial) and Type 2 (or parallel) singularities.
}

\section{ORIENTATION REPRESENTATION}

A novel three-angle orientation representation, later called the Tilt-and-Torsion (T\&T) angles, was proposed in [12] in 1999, in conjunction with a new method for computing the orientation workspace of symmetric spatial parallel mechanisms. It was shown that the T\&T angles take full advantage of a mechanism's symmetry. These angles were also independently introduced in [13] and [14] in 1999. Later, it was found out that the angles had been proposed in [15] in 1984 under the name halfplanedeviation-twist angles. The author of that reference proposed the set due to its indisputable advantages in modeling the limits of human body joints. Yet, again in 1999, these angles were proposed independently in [16] as a new standard in modeling angular joint motion, and particularly that of the spinal column's vertebra. These angles are also used for computer animation of articulated bodies, known as the swing-and-twist representation. Finally, in [17], the advantages of the T\&T angles in the study of spatial parallel mechanisms were further demonstrated. It was shown that there is a class of 3-DOF mechanisms that have always a zero torsion.

The T\&T angles are defined in two stages - a tilt and a torsion. This does not, however, mean that only two angles define the T\&T angles but simply that the axis of tilt is variable and is defined by another angle. In the first stage,+ illustrated in Fig. 1(a), the body frame is tilted about a horizontal axis, $a$, at an angle $\theta$, referred to as the tilt. The axis $a$ is defined by an angle $\phi$, called the azimuth, which is the angle between the projection of the body $z^{\prime}$ axis onto the fixed $x y$ plane and the fixed $x$ axis. In the second stage, illustrated in Fig. 1(b), the body frame is rotated about the body $z^{\prime}$ axis at an angle $\sigma$, called the torsion.

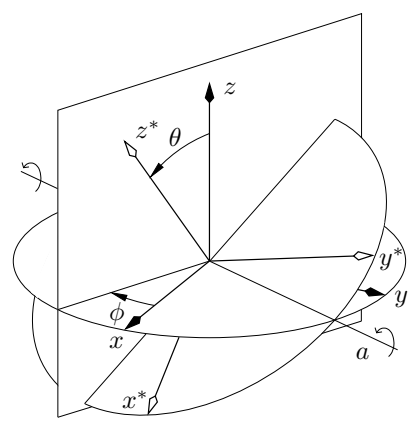

(a)

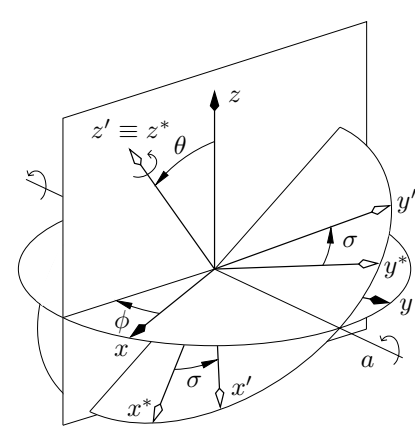

(b)
Fig. 1. The successive rotations of the T\&T angles: (a) tilt, (b) torsion. 
For space limitations, we will omit the otherwise quiteinteresting details of the derivation process (see [17]), and write directly the resulting rotation matrix of the T\&T angles, which is

$$
\begin{aligned}
& \mathbf{R}(\phi, \theta, \sigma)= \\
& {\left[\begin{array}{ccc}
c_{\phi} c_{\theta} c_{\sigma-\phi}-s_{\phi} s_{\sigma-\phi} & -c_{\phi} c_{\theta} s_{\sigma-\phi}-s_{\phi} c_{\sigma-\phi} & c_{\phi} s_{\theta} \\
s_{\phi} c_{\theta} c_{\sigma-\phi}+c_{\phi} s_{\sigma-\phi} & -s_{\phi} c_{\theta} s_{\sigma-\phi}+c_{\phi} c_{\sigma-\phi} & s_{\phi} s_{\theta} \\
-s_{\theta} c_{\sigma-\phi} & s_{\theta} s_{\sigma-\phi} & c_{\theta}
\end{array}\right],}
\end{aligned}
$$

where $c_{\phi}=\cos \phi, s_{\phi}=\sin \phi, c_{\theta}=\cos \theta, s_{\theta}=\sin \theta$, $c_{\sigma-\phi}=\cos (\sigma-\phi)$ and $s_{\sigma-\phi}=\sin (\sigma-\phi)$.

One of the properties of three-angle orientation representation is that a given orientation can be represented by at least two triplets of angles. To avoid this and the representational singularity at $\theta=\pi$ (which is hardly achieved by any parallel mechanism), we set the ranges of the azimuth, tilt, and torsion as, respectively, $\phi \in(-\pi, \pi]$, $\theta \in[0, \pi)$, and $\sigma \in(-\pi, \pi]$. Then, probably the most valuable property of the $T \& \mathrm{~T}$ angles is that for the ranges just defined, the angles $(\theta, \phi, \sigma)$ can be represented in a cylindrical coordinate system $(r, \phi, h)$ through a one-to-one mapping. In other words, any orientation (except a $\theta=\pi$ one) corresponds to a unique point within a cylinder in the cylindrical coordinate system, and vice versa. The reason is that the $\mathrm{T} \& \mathrm{~T}$ representational singularity at $\theta=0$ is of the same nature as the singularity of the cylindrical coordinate system occurring at zero-radius $(r=0)$.

Fig. 2(a) shows an example of the orientation workspace of a symmetric spatial parallel mechanism, and illustrates the use of the T\&T angles in a cylindrical coordinate system [12]. In cases where the torsion is irrelevant, as in a parallel kinematic machine with an axisymmetric tool, we are only interested in the set of all possible directions of the body $z^{\prime}$ axis (all $\phi$ and $\theta$ ), referred to as the projected orientation workspace. An example of the latter is shown in Fig. 2(b). We will use the same polar format to represent the singularity loci of spherical parallel mechanisms for a constant torsion, in analogy to the constant-orientation singularity plots of planar parallel mechanisms.

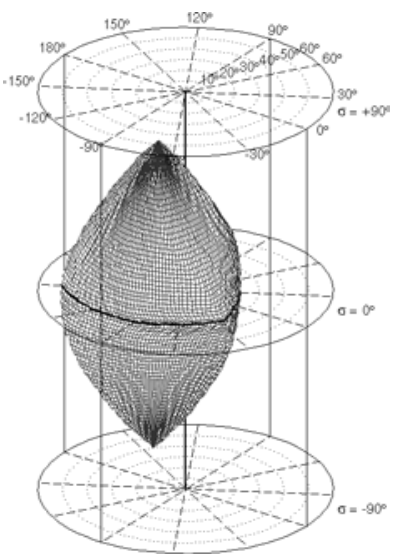

(a)

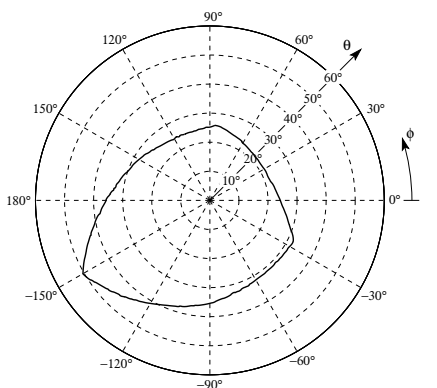

(b)
Fig. 2. Example of (a) the orientation workspace and (b) the projected orientation workspace of a symmetric spatial parallel mechanism.

\section{SingULARITY LOCI OF 3-UPPS/S SPMS}

Consider now the 3-DOF $3-U \underline{P S} / S$ symmetric SPM shown in Fig. 3, which is the simplest spherical parallel mechanism, from any point of view. It consists of a mobile platform connected to a base through a spherical joint and three identical UPS legs, each comprising a prismatic actuator ending by a universal joint and a spherical joint. Let the centers of the base and platform joints be denoted by $O_{i}$ and $B_{i}$, respectively (in this paper, $i=1,2,3$ ). Let $O-x y z$ be the frame that is fixed to the base (base frame) and $C-x^{\prime} y^{\prime} z^{\prime}$ be the frame that is fixed to the mobile platform (mobile frame), and let $O \equiv C$ coincide with the center of the spherical joint that connects the mobile platform to the base. Finally, let these frames be chosen in such a manner that the vectors connecting $O$ with $O_{i}$ and expressed in the base frame, denoted by $\mathbf{r}_{O_{i}}$, and the ones connecting $O$ with $B_{i}$ and expressed in the mobile frame, denoted by $\mathbf{r}_{B_{i}}^{\prime}$, be

$$
\begin{aligned}
& \mathbf{r}_{O_{i}}=\left[\begin{array}{c}
\ell_{1} \cos \left((i-1) \frac{2 \pi}{3}\right) \sin \beta_{1} \\
\ell_{1} \sin \left((i-1) \frac{2 \pi}{3}\right) \sin \beta_{1} \\
-\ell_{1} \cos \beta_{1}
\end{array}\right], \\
& \mathbf{r}_{B_{i}}^{\prime}=\left[\begin{array}{c}
\ell_{2} \cos \left((i-1) \frac{2 \pi}{3}\right) \sin \beta_{2} \\
\ell_{2} \sin \left((i-1) \frac{2 \pi}{3}\right) \sin \beta_{2} \\
\ell_{2} \cos \beta_{2}
\end{array}\right],
\end{aligned}
$$

where $\ell_{1}$ and $\ell_{2}$ are the lengths of the vectors, $\beta_{1}$ is the angle between $\mathbf{r}_{O_{i}}$ and the negative base $z$ axis, and $\beta_{2}$ is the angle between $\mathbf{r}_{B_{i}}^{\prime}$ and the mobile $z^{\prime}$ axis. Obviously, $\beta_{2}$ should be non-zero if we want our mechanism to have all of its three degrees of freedom controllable. In addition, to alleviate the presentation, we will make the natural assumption that $0 \leq \beta_{1} \leq \pi / 2$ and $0<\beta_{2} \leq \pi / 2$.

Skipping the details, the input-output velocity equation for our mechanism can be easily derived as

$$
\mathbf{Z} \boldsymbol{\omega}=\left[\begin{array}{c}
\left(\mathbf{r}_{O_{1}} \times \mathbf{r}_{B_{1}}\right)^{T} \\
\left(\mathbf{r}_{O_{2}} \times \mathbf{r}_{B_{2}}\right)^{T} \\
\left(\mathbf{r}_{O_{3}} \times \mathbf{r}_{B_{3}}\right)^{T}
\end{array}\right] \boldsymbol{\omega}=\operatorname{diag}\left(\rho_{1}, \rho_{2}, \rho_{3}\right) \dot{\boldsymbol{\rho}}
$$

where $\boldsymbol{\omega}$ is the vector of angular velocity of the platform and $\boldsymbol{\rho}=\left[\rho_{1}, \rho_{2}, \rho_{3}\right]^{T}$ is the vector of leg lengths.

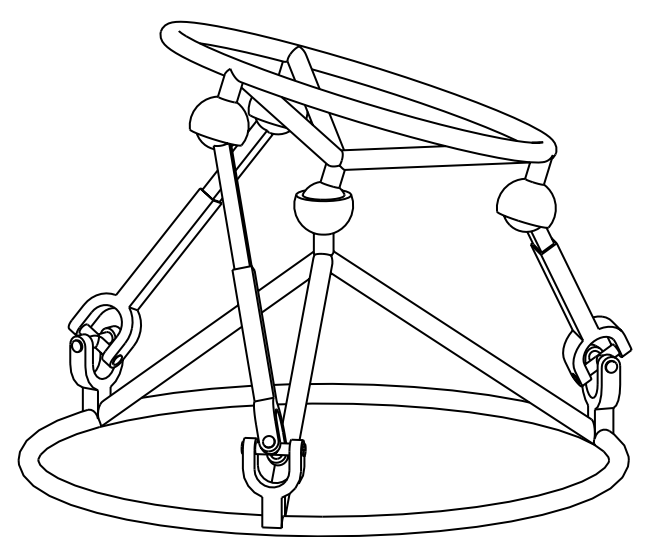

Fig. 3. A 3-UPS/S symmetric spherical parallel mechanism. 


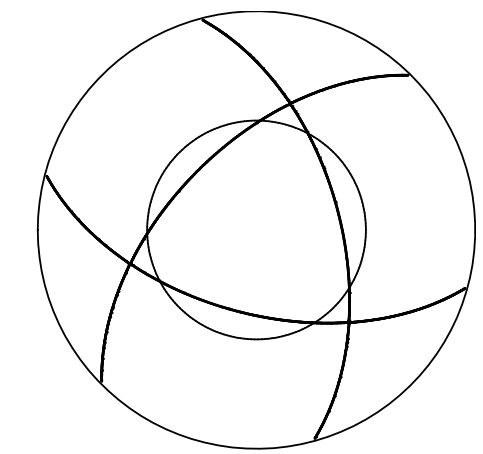

(a) $\beta_{1}=54.74^{\circ}, \beta_{2}=54.74^{\circ}, \sigma=30^{\circ}$

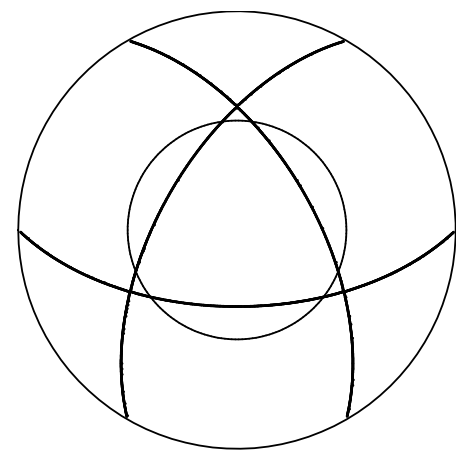

(b) $\beta_{1}=54.74^{\circ}, \beta_{2}=54.74^{\circ}, \sigma=60^{\circ}$

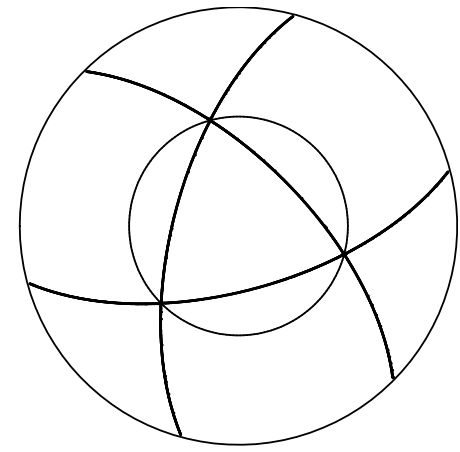

(c) $\beta_{1}=54.74^{\circ}, \beta_{2}=54.74^{\circ}, \sigma=90^{\circ}$

Fig. 4. Example of the singularity loci of a $3-U \underline{P} S / S$ spherical parallel mechanism.

The Type 1 singularity loci of this and other SPMs will be the subject of another (even more technical) paper. Indeed, while the geometric interpretation of Type 1 singularity loci is trivial, the computation of these loci is a challenging task. However, let us only note that, unlike the common practice, these singularities cannot be generally found from the above velocity equation. The Type 2 singularities, on the other hand, occur when the matrix $\mathbf{Z}$ is singular. Now, the key idea of our paper is to use the T\&T angles to express $\mathbf{r}_{B_{i}}^{\prime}$ in the base frame, i.e., $\mathbf{r}_{B_{i}}=\mathbf{R r}_{B_{i}}^{\prime}$, where $\mathbf{R}$ is as given in (1). Then, after some astute trigonometric manipulations, the equation defining the Type 2 singularities becomes:

$$
\begin{aligned}
\Delta & =\operatorname{det} \mathbf{Z} \\
& =\ell_{1}^{3} \ell_{2}^{3} \frac{3 \sqrt{3}}{16} \sin \beta_{1} \sin \beta_{2}\left(p_{1} \sin 3 \phi+p_{2} \cos 3 \phi+p_{3}\right) \\
& =\ell_{1}^{3} \ell_{2}^{3} \frac{3 \sqrt{3}}{16} \sin \beta_{1} \sin \beta_{2} \Delta^{\prime}=0,
\end{aligned}
$$

where

$p_{1}=(1-\cos \theta) \sin \theta\left(c_{1} \cos 2 \sigma \sin 2 \beta_{2}-c_{2} \cos \sigma \sin 2 \beta_{1}\right)$, $p_{2}=(1-\cos \theta) \sin \theta \sin \sigma\left(c_{2} \sin 2 \beta_{1}-2 c_{1} \cos \sigma \sin 2 \beta_{2}\right)$, $p_{3}=(1+\cos \theta) \sin \sigma\left((1+\cos \theta) \cos \sigma \sin 2 \beta_{1} \sin 2 \beta_{2}+\right.$ $\left.2 c_{1} c_{2} \cos \theta+12 c_{1}+12 c_{2}-10 c_{1} c_{2}-16\right)$,

and

$$
c_{j}=1+\cos ^{2} \beta_{j}=\left(3+\cos 2 \beta_{j}\right) / 2,
$$

for $j=1,2$.

We will not miss this opportunity to stress on the importance of the manipulations that led us to the above simple equation. Because of the use of the T\&T angles and the symmetry of the mechanism, it is logical to expect that for a constant torsion, the singularity loci will repeat themselves with a period of $120^{\circ}$ in $\phi$. This is an indication that the expression for these Type 2 singularity loci may possibly be written in terms of $3 \phi$-and indeed it can. Without this simplification, we will not be dealing with a linear trigonometric equation in $\sin 3 \phi$ and $\cos 3 \phi$ but with a cubic one in $\sin \phi$ and $\cos \phi$. Similarly, though this is of much lesser importance, the singularities will be exactly the same even if $\beta_{j}$ were replaced by $\beta_{j}+180^{\circ}(j=1,2)$. This means that the Type 2 singularity expression may probably be written in terms of $2 \beta_{1}$ and $2 \beta_{2}$, which makes the expression more compact and, therefore, easier to analyze.

Now, we can proceed with analyzing the Type 2 singularity loci and finding a way to plot them. Obviously, when $\beta_{1}=0$ or $\beta_{2}=0$, we have an architecture singularity and the resulting mechanism is singular for any orientation. Rejecting these trivial solutions, we need to solve the linear polynomial equation $\Delta^{\prime}=0$ in $\sin 3 \phi$ and $\cos 3 \phi$. We may substitute with the tangent-half-angle identities and receive two solutions for $3 \phi$. These solutions are functions of $\theta$ and $\sigma$ (and $\beta_{1}$ and $\beta_{2}$, of course). Thus, for a constant $\sigma$, we obtain two parametric functions for the curves of interest. Each of these functions can be readily plotted by incrementing $\theta$ from 0 to $180^{\circ}$ and plotting the corresponding three values for $\phi$ (spaced apart by $120^{\circ}$ ).

An important observation can be made if we substitute $\sigma=0$ in $\Delta^{\prime}$ to obtain:

$$
(1-\cos \theta) \sin \theta\left(c_{1} \sin 2 \beta_{2}-c_{2} \sin 2 \beta_{1}\right) \sin 3 \phi=0 .
$$

From the above, it follows that whatever the design of the mechanism, there will be a singularity at $\phi=n 60^{\circ}(n=$ $1,2, \ldots, 6)$ for any $\theta$. If also $\beta_{1}=\beta_{2}$, then there will be a singularity for any direction (any $\theta$ and $\phi$ ). The same occurs for $\sigma=180^{\circ}$. Therefore, one should always use the mechanism far from the zero or $180^{\circ}$ torsion.

Figs. 4 and 5 show the singularity loci of two different 3-U $\underline{P} S / S$ SPMs, for three different torsions. The plots are in our constant-torsion polar format illustrated in Fig. 2(b). To simplify the representation, however, the polar grid is replaced by only two concentric circles corresponding to $\theta=90^{\circ}$ (for the inner one) and $\theta=180^{\circ}$.

The maximum tilt angle that can be achieved in any direction within the range $\sigma \in\left[30^{\circ}, 90^{\circ}\right]$, without reaching a singularity, is about $50^{\circ}$ for the first mechanism (the base as well as the platform vectors are orthogonal), and $70^{\circ}$ for the second one $\left(\beta_{1}=30^{\circ}, \beta_{2}=90^{\circ}\right)$. Indeed, from a Type 2 singularity point of view, the second mechanism represents one of the most optimal designs. Note, that the singularity loci of the first mechanism can be found in a trivial manner using the $Z Y X$ Euler angles and coordinate frames coinciding with vectors $\mathbf{r}_{B_{i}}$ and $\mathbf{r}_{O_{i}}$ [18]. 


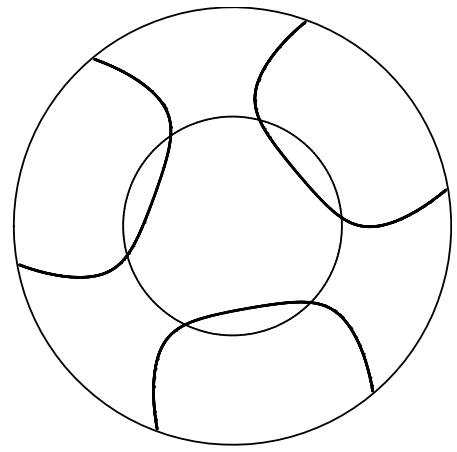

(a) $\beta_{1}=30^{\circ}, \beta_{2}=90^{\circ}, \sigma=30^{\circ}$

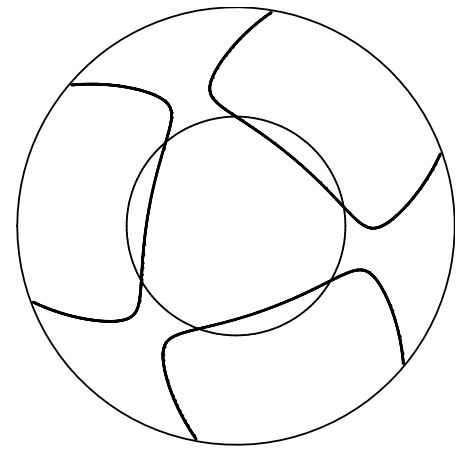

(b) $\beta_{1}=30^{\circ}, \beta_{2}=90^{\circ}, \sigma=60^{\circ}$

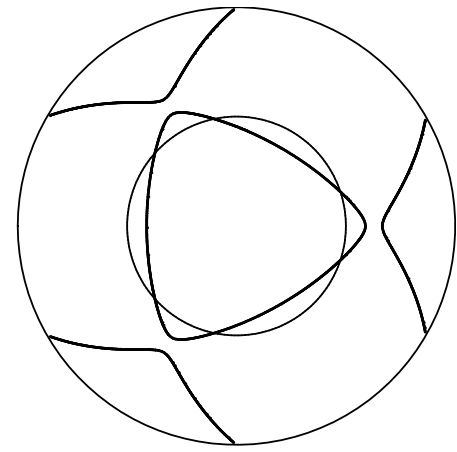

(c) $\beta_{1}=30^{\circ}, \beta_{2}=90^{\circ}, \sigma=90^{\circ}$

Fig. 5. Example of the singularity loci of a $3-U \underline{P} S / S$ spherical parallel mechanism.

\section{Singularity LOCI OF 3-RRR SPMS}

Consider now the 3-DOF 3- $\underline{R} R R$ symmetric SPM shown in Fig. 6. It consists of three identical $\underline{R} R R$ legs in which the base joint is actuated. The axes of all nine revolute joints intersect at one point. Let the axes of the base, intermediate, and platform joints be defined by the unit vectors $\mathbf{r}_{O_{i}}, \mathbf{r}_{A_{i}}$, and $\mathbf{r}_{B_{i}}$, respectively. Let $O-x y z$ be the base frame and $C-x^{\prime} y^{\prime} z^{\prime}$ be the mobile frame, and let $O \equiv C$ coincide with the intersection point of all joint axes. Finally, let these frames be chosen in such a manner that the vectors $\mathbf{r}_{O_{i}}$ expressed in the base frame and the vectors $\mathbf{r}_{B_{i}}^{\prime}$ expressed in the mobile frame be the same as the ones given by (2) and (3), but with $\ell_{1}=\ell_{2}=1$. Naturally, $\beta_{1}$ and $\beta_{2}$ will have the same geometric definition as before.

Skipping again the details, the input-output velocity equation for this mechanism can be easily derived as

$$
\begin{array}{r}
\mathbf{Z} \boldsymbol{\omega}=\left[\begin{array}{c}
\left(\mathbf{r}_{A_{1}} \times \mathbf{r}_{B_{1}}\right)^{T} \\
\left(\mathbf{r}_{A_{2}} \times \mathbf{r}_{B_{2}}\right)^{T} \\
\left(\mathbf{r}_{A_{3}} \times \mathbf{r}_{B_{3}}\right)^{T}
\end{array}\right] \boldsymbol{\omega}=\operatorname{diag}\left(\left(\mathbf{r}_{A_{1}} \times \mathbf{r}_{B_{1}}\right)^{T} \mathbf{r}_{O_{1}},\right. \\
\left.\quad\left(\mathbf{r}_{A_{2}} \times \mathbf{r}_{B_{2}}\right)^{T} \mathbf{r}_{O_{2}},\left(\mathbf{r}_{A_{3}} \times \mathbf{r}_{B_{3}}\right)^{T} \mathbf{r}_{O_{3}}\right) \dot{\boldsymbol{\theta}}
\end{array}
$$

where $\boldsymbol{\theta}$ is the vector of active-joint variables.

From the above equation, we can easily see that the mechanism is at a Type 2 singularity when the normals to the planes of the distal links are linearly dependent. However, for a given orientation of the mobile platform, there

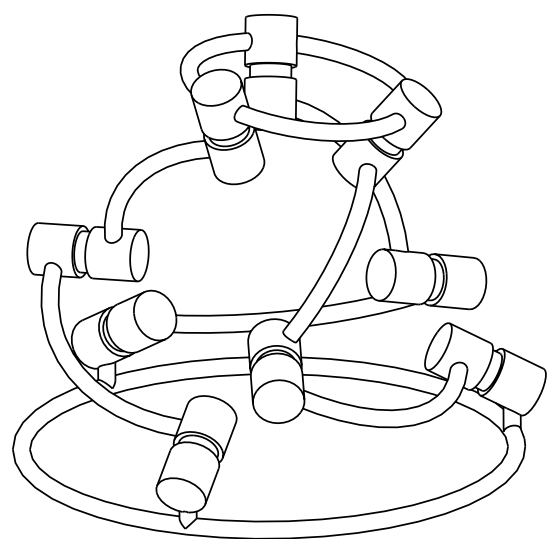

Fig. 6. A 3- $R R R$ symmetric spherical parallel mechanism. are generally two possibilities for each vector $\mathbf{r}_{A_{i}}$. (Note that the 3-RRR SPM has eight working modes, i.e., eight solutions to its inverse kinematics.) Thus, similarly to the much simpler case of $3-\underline{R R R}$ planar parallel mechanisms studied in [19], the determinant of $\mathbf{Z}$ will contain three different radicals. It is virtually impossible to study such an expression using an analytic method. Hence, the only way to plot the Type 2 singularity loci of general $3-\underline{R} R R$ spherical parallel mechanisms will be through a numerical or a discretization method.

Unlike for planar 3- $\underline{R} R R$ parallel mechanisms, however, there is an easy way to significantly simplify the kinematics of 3-RRR SPMs: it suffices to use links of $90^{\circ}$. One of the nice properties that have such mechanisms is that their workspace is (theoretically) unlimited. The other is that for a given orientation of the mobile platform, the two solutions for each vector $\mathbf{r}_{A_{i}}$ are opposite in direction and the equation for $\mathbf{r}_{A_{i}}$ simplifies to:

$$
\mathbf{r}_{A_{i}}= \pm\left(\mathbf{r}_{O_{i}} \times \mathbf{r}_{B_{i}}\right) .
$$

Thus, not only the Type 2 singularity loci become the same for all working modes, but the expression defining these singularities becomes significantly simplified and with no radicals at all. Indeed, using the T\&T angles and the same reasoning as in the previous section, we can obtain that the equation defining the Type 2 singularity loci for $3-\underline{R} R R$ spherical parallel mechanisms with links of $90^{\circ}$ is:

$$
\begin{aligned}
\Delta=\operatorname{det} \mathbf{Z}= \pm \frac{3 \sqrt{3}}{32} \sin \beta_{2}\left(p_{1} \sin 3 \phi \cos 3 \phi+\right. \\
\left.\quad p_{2} \sin ^{2} 3 \phi+p_{3} \sin 3 \phi+p_{4} \cos 3 \phi+p_{5}\right) \\
= \pm \frac{3 \sqrt{3}}{32} \sin \beta_{2} \Delta^{\prime}=0
\end{aligned}
$$

where $p_{1}, p_{2}, \ldots, p_{5}$ are coefficients depending only on $\beta_{1}$, $\beta_{2}, \sigma$, and $\theta$. While relatively small, these coefficients will still fill up a page and will therefore not be presented here.

Once again, we substitute in the above equation the tangent-half-angle identities and obtain four solutions for $3 \phi$. These solutions are functions of $\theta$ and $\sigma$ (and $\beta_{1}$ and $\beta_{2}$ ). Thus, for a constant $\sigma$, we obtain four parametric singularity curves. Each of these curves can be displayed by incrementing $\theta$ from 0 to $180^{\circ}$ and plotting the corresponding three values for $\phi$ (spaced apart by $120^{\circ}$ ). 


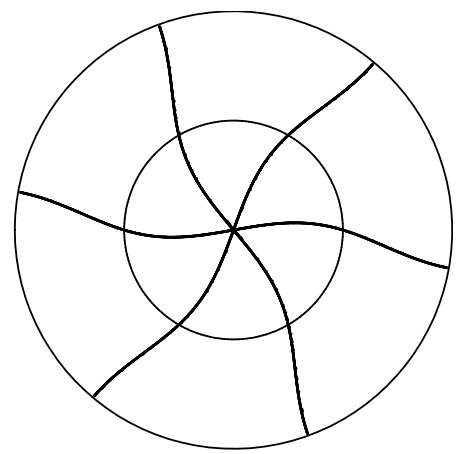

(a) $\beta_{1}=90^{\circ}, \beta_{2}=90^{\circ}, \sigma=30^{\circ}$

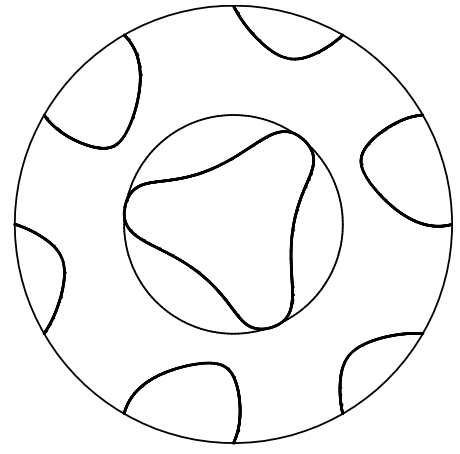

(d) $\beta_{1}=90^{\circ}, \beta_{2}=54.74^{\circ}, \sigma=30^{\circ}$

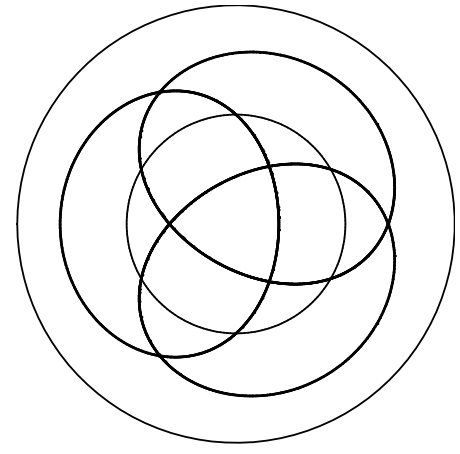

(b) $\beta_{1}=0^{\circ}, \beta_{2}=54.74^{\circ}, \sigma=0^{\circ}$

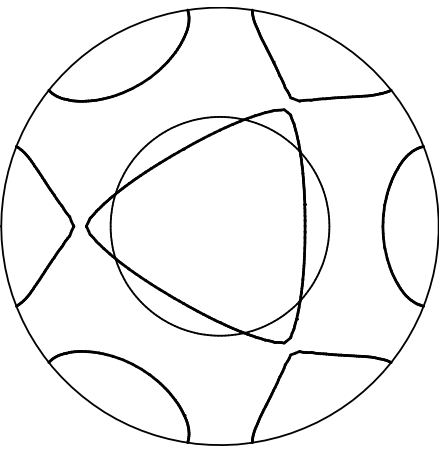

(e) $\beta_{1}=80^{\circ}, \beta_{2}=30^{\circ}, \sigma=0^{\circ}$

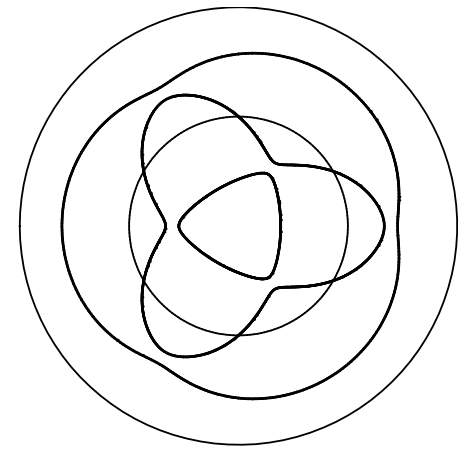

(c) $\beta_{1}=0^{\circ}, \beta_{2}=60^{\circ}, \sigma=0^{\circ}$

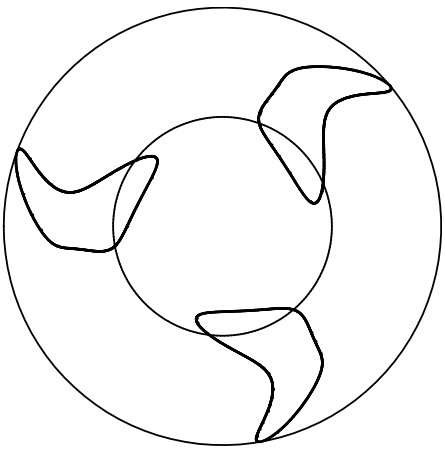

(f) $\beta_{1}=45^{\circ}, \beta_{2}=45^{\circ}, \sigma=20^{\circ}$

Fig. 7. Examples of the singularity loci of several $3-\underline{R} R R$ spherical parallel mechanisms.

Before presenting a couple of examples, let us take advantage of our analytic expression and try to find out what designs could lead to simplified or particular cases. We see immediately that if $\beta_{2}=0$, then we have an architecture singularity (the platform can freely rotate about the coincident platform joint axes). If, on the other hand, we have $\beta_{1}=0$, our mechanism becomes, as expected, significantly simplified. Indeed, if $\beta_{1}=0$, then we can find that (9) simplifies to

$$
\begin{aligned}
& \cos (3 \phi-3 \sigma)= \\
& \frac{\cos \theta\left(1+3 \cos ^{4} \beta_{2}+\cos ^{2} \theta\left(1-\cos ^{4} \beta_{2}+4 \cos ^{2} \beta_{2}\right)\right)}{\sin ^{3} \theta \sin \beta_{2} \cos \beta_{2}\left(1+\cos ^{2} \beta_{2}\right)} .
\end{aligned}
$$

This means that the Type 2 singularity loci of $3-\underline{R R R}$ SPMs with coaxial base joints and $90^{\circ}$ links have the same shape for any $\sigma$-changing $\sigma$ only rotates the loci.

Fig. 7 shows the singularity loci of several different 3-RRR SPMs. Once again, the two concentric circles correspond to $\theta=90^{\circ}$ and $\theta=180^{\circ}$. Fig. 7(a) shows the singularity loci of a $3-\underline{R} R R$ SPM with coplanar base and platform joint axes, shown in Fig. 8. For any torsion angle, the Type 2 singularity loci of this mechanism are three equally-spaced identical curves that pass through the center, $\theta=0$. SPMs of this type, but not necessarily with $90^{\circ}$ links (which are hardly of any use due to the omnipresence of singularities), were studied in [5].

Figs. 7(b) and 7(c) correspond to two designs with coincident base joint axes. As we proved a few paragraphs back, the Type 2 singularity loci of such designs are identical for any torsion angle, but only rotated at the torsion angle. It can be shown that the largest minimum tilt angle occurs in the design where the three platform axes are orthogonal (i.e., $\beta_{2}=\cos ^{-1}(1 / \sqrt{3}) \approx 54.74^{\circ}$ ). However, this largest minimum tilt angle is only about $35^{\circ}$. Therefore, 3- $\underline{R} R R$ spherical parallel mechanisms with $90^{\circ}$ links and coincident base joint axes have a relatively small singularity-free workspace. Yet, a US patent was recently filed on this design [20], though not insisting on $90^{\circ}$ links, and a prototype was developed for laparoscopic surgery. It is interesting to mention, though, that if $\beta_{2}=90^{\circ}$, $\Delta^{\prime}=-4 \cos \theta \sin ^{2} \theta$, which means that this design has a Type 2 singularity only when $\theta=0,90^{\circ}$, or $180^{\circ}$. According to [4], such a spherical wrist was developed by the Japanese company Shin Meiwa Industry Ltd., but with proximal links of $70^{\circ}$ and distal links of $95^{\circ}$. Reference [4] states, however, that the "kinematic and static performance of this wrist is very poor" and suggests to rather use links of $50^{\circ}$ and $105^{\circ}$.

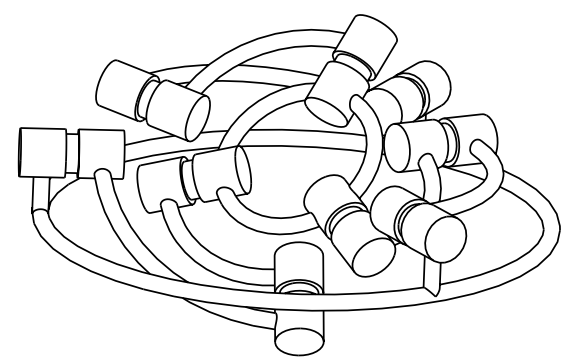

Fig. 8. A 3-RRR symmetric SPM with coplanar base and platform axes. 


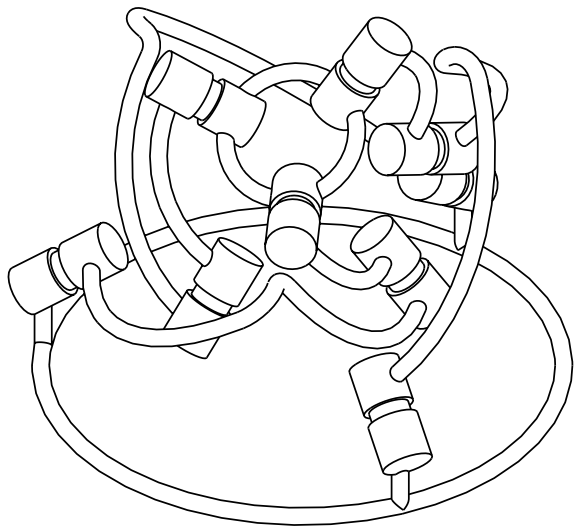

Fig. 9. The Agile Eye-the least singularity-prone 3-RRR SPM.

Fig. 7(d) corresponds to what might seem to be a special design, as the base axes are coplanar while the platform axes are orthogonal. However, we can see that apart from the fancy shape of the singularity loci, there is nothing particular about this design and it does not have a large singularity-free workspace.

Fig. 7(e), on the other hand, corresponds to what looks like an ordinary design. Yet, this mechanism can achieve, without crossing a Type 2 singularity, tilt angles of up to $70^{\circ}$ in any direction and with torsion angles of up to $\pm 85^{\circ}$. Not surprisingly, a haptic device corresponding to this design has been already developed [21].

Finally, Fig. 7(f) is one of a series of examples demonstrating that for virtually any torsion when both $\beta_{1}$ and $\beta_{2}$ approach $54.74^{\circ}$ (the Agile Eye design [7] shown in Fig. 9), the Type 2 singularity loci diminish to finally disappear (in fact, reduce to three points). Indeed, it was shown in [8] that, in the Agile Eye, the Type 2 singularity loci coincide with the Type 1 ones, which are only a couple of curves in the whole orientation workspace. Thus, although with no formal proof, we can safely state that the least singularityprone SPM is the Agile Eye.

\section{Conclusion}

We showed in this paper that the Tilt-and-Torsion angles are a powerful geometric tool for the analysis of symmetric spherical parallel mechanisms. Particularly, we derived simple expressions for the Type 2 singularity loci of two of the most basic spherical parallel mechanisms and studied several designs of interest. Naturally, examples of the singularity loci were given. It is clear that other properties of symmetric spherical parallel mechanisms can be studied with the same success using our approach.

\section{ACKNOWLEDGMENT}

This work was supported by the Natural Sciences and Engineering Research Council of Canada (NSERC), the Fonds de recherche sur la nature et les technologies of Quebec (FQRNT), and the Canada Research Chair Program. The authors would also like to thank Mr. Frédéric Pelletier for creating the CAD models shown in this paper.

\section{REFERENCES}

[1] Di Gregorio, R., "Kinematics of a new spherical parallel manipulator with three equal legs: The 3-URC wrist," Journal of Robotic Systems, Vol. 18, No. 5, pp. 213-219, 2001.

[2] Karouia, M., and Hervé, J.M., "A family of novel orientational 3-DOF parallel robots," RoManSy 14, pp. 359-368, Springer, 2002.

[3] Kong, X., and Gosselin, C.M., "Type synthesis of 3-DOF spherical parallel manipulators based on screw theory," Proceedings of the ASME 2002 Design Engineering Technical Conferences, Montreal, Canada, September 29 - October 2, 2002.

[4] Asada, H., and Cro Granito, J.A., "Kinematic and static characterization of wrist joints and their optimal design," Proceedings of the IEEE International Conference on Robotics and Automation, St. Louis, MO, Vol. 2, pp. 244-250, March 25-28, 1985.

[5] Gosselin, C.M., and Angeles, J., "The optimum kinematic design of a spherical three-degree-of-freedom parallel manipulator," Journal of Mechanisms, Transmissions, and Automation in Design, Vol. 111, pp. 202-207, June 1989.

[6] Innocenti, C., and Parenti-Castelli, V., "Echelon form solution of direct kinematics for the general fully-parallel spherical wrist," Mechanism and Machine Theory, Vol. 28, No. 4, pp. 553-561, 1993.

[7] Gosselin, C.M., and Hamel, J.-F, "The Agile Eye: A highperformance three-degree-of-freedom camera-orienting device," Proceedings of the IEEE International Conference on Robotics and Automation, San Diego, CA, pp. 781-786, 1994.

[8] Gosselin, C.M., and Wang, J., "Singularity loci of a special class of spherical three-degree-of-freedom parallel mechanisms with revolute actuators," The International Journal of Robotics Research, Vol. 21, No. 7, pp. 649-659, 2002.

[9] Gosselin, C.M., and Angeles, J., "Singularity analysis of closed-loop kinematic chains," IEEE Transactions on Robotics \& Automation, Vol. 6, No. 3, pp. 281-290, 1990.

[10] Sefrioui, J., and Gosselin, C.M., "Étude et représentation des lieux de singularité des manipulateurs parallèles sphériques à trois degrés de liberté avec actionneurs prismatiques," Mechanism and Machine Theory, Vol. 29, No. 4, pp. 559-579, 1994.

[11] Alici, G., and Shirinzadeh, B., "Topology optimization and singularity analysis of a 3-SPS parallel manipulator with a passive constraining spherical joint," Mechanism and Machine Theory, Vol. 39, No. 2, pp. 215-235, 2004.

[12] Bonev, I.A., and Ryu, J., "Orientation workspace analysis of 6-DOF parallel manipulators," Proceedings of the ASME 1999 Design Engineering Technical Conferences, Las Vegas, NV, 1999.

[13] Huang, T., Wang, J., and Whitehouse, D.J., "Closed form solution to workspace of hexapod-based virtual axis machine tools," Journal of Mechanical Design, Vol. 121, pp. 26-31, March 1999.

[14] Wang, Y., "Workspace analysis of a novel closed-chain manipulator," Thesis, Case Western Reserve Univ., Cleveland, OH, 1999.

[15] Korein, J.U., A Geometric Investigation of Reach, MIT Press, 1984.

[16] Crawford, N.R., Yamaguchi, G.T., and Dickman, C.A., "A new technique for determining 3-D joint angles: The tilt/twist method," Clinical Biomechanics, Vol. 14, No. 3, pp. 153-165, 1999.

[17] Bonev, I.A., Zlatanov, D., and Gosselin, C.M., "Advantages of the modified Euler angles in the design and control of PKMs," 2002 Parallel Kinematic Machines International Conference, Chemnitz, Germany, pp. 171-188, April 23-25, 2002.

[18] Wang, J., and Gosselin, C.M., "Singularity loci of a special class of spherical 3-DOF parallel mechanisms with prismatic actuators," Journal of Mechanical Design, Vol. 126, pp. 319-325, 2004.

[19] Bonev, I.A., and Gosselin, C.M., "Singularity loci of planar parallel manipulators with revolute joints," 2nd Workshop on Computational Kinematics, Seoul, South Korea, May 20-22, 2001.

[20] Payandeh, S., Li, T., and Van Der Wal, H., "Devices for positioning implements about fixed points," US Patent Application Publication No. US 2004/0024387, published on February 5, 2004.

[21] Takeda, Y., Funabashi, H. and Sasaki, Y., "Development of a spherical in-parallel actuated mechanism with three degrees of freedom with large working space and high motion transmissibility," JSME International Journal, Vol. 39, No. 3, pp. 541-548, 1996. 